\title{
Horizons/Théâtre
}

Revue d'études théâtrales

\section{Transformation du politique dans le théâtre négro- africain d'expression française des débuts à nos jours}

\section{Abdelillah Krim}

\section{(2) OpenEdition Journals \\ Édition électronique \\ URL : https://journals.openedition.org/ht/1071 \\ DOI : 10.4000/ht.1071 \\ ISSN : 2678-5420 \\ Éditeur \\ Presses universitaires de Bordeaux}

\section{Édition imprimée}

Date de publication : 31 décembre 2018

Pagination : 78-94

ISBN : 979-10-300-0318-5

ISSN : 2261-4591

Référence électronique

Abdelillah Krim, «Transformation du politique dans le théâtre négro-africain d'expression française des débuts à nos jours », Horizons/Théâtre [En ligne], 13| 2018, mis en ligne le 01 janvier 2019,

consulté le 17 mai 2022. URL : http://journals.openedition.org/ht/1071 ; DOI : https://doi.org/10.4000/ ht.1071 


\section{AbDelillah Krim}

Abdelillah Krim est actuellement en troisième année d'études doctorales à l'Université Mohammed V de Rabat au sein du laboratoire « Langues, Littératures, Arts et culture ». II prépare une thèse sur "La métaphore de la marge dans l'œuvre dramatique de Bernard-Marie Koltès » sous la direction de Hassan Moustir. II a soutenu en octobre 2014 un mémoire de master intitulé "Fantomachie de Valère Novarina. Le méta-drame : un au-delà du théâtre ». Il est aussi l'auteur d'un article intitulé " Théâtre de l'empêchement, théâtre de l'ab-sens : mise en scène du vide dans l'œuvre de Valère Novarina » (dans Le Théâtre aujourd'hui : écriture et art de la performance). D'autres articles sont en cours de publication au Maroc et à l'étranger. Il est actuellement titulaire d'une bourse d'excellence du Centre National de la Recherche Scientifique et Technique (CNRST). En 2011, il a pu effectuer un séjour d'étude en France d'un an, à l'Université Paul Valéry, Montpellier III, grâce à une bourse d'excellence de la commission européenne (ERASMUS MUNDUS - Averroès).

Mail: krim.abdelillah@outlook.fr

Résumé : Le théâtre africain ne peut-il être que politique? Voilà la question que suscite, en Afrique, I'histoire d'une discipline que l'Occident a pendant longtemps tenu à instituer, non seulement comme une propriété exclusive, mais aussi comme le fruit béni de la réussite de sa mission civilisatrice. Néanmoins, les dramaturges africains d'expression française réussiront à transformer la première rencontre de l'homme noir avec le théâtre pontin, prototype traditionnel stigmatisant, en autant de véhéments plaidoyers pour un retour aux heures de gloire du continent. Des premières exploita-

Abstract : Could African theater be only political? That is the question that emerges from the history of a discipline that the West has long wanted to establish. Not only as an exclusive property, but also as the blessed fruit of its civilizing mission. However, French-speaking African playwrights will transform the first encounter of black men with the "théâtre pontin" - a traditional stigmatizing prototype - into many vehement advocacies and nostalgic eulogies of the revival of the glorious days of the continent. tions du roi Chaka aux modèles identitaires hybrides de la génération des années 1990, qui continue à produire jusqu'à aujourd'hui, le politique agit comme un vecteur d'affirmation identitaire comme il avait été un support d'insoumission. Plus qu'un réflexe d'auto-défense, le politique orne ce théâtre d'un habit d'actualité qui l'inscrit directement dans les grandes questions de son époque.

Mots-clés : théâtre d'Afrique subsaharienne, politique, théâtre historique, identité.

From the first exploitations of King Chaka to the hybrid identity of the writers of the 90 s generation, politics act as a vector of identity affirmation, as it has been a support for rebellion. More than a reflex of self-defense, politics give this theater a meaning of contemporaneity which enrolls it directly in the great questions of its time.

KeYwords: Sub-saharan african theater, politics, historic theater, identity. 


\section{Transformation du politique dans le théâtre négro-africain d'expression française des débuts à nos jours}

$\ll$ Et de même que la peste, Le théâtre est fait pour vider collectivement des abcès. » Antonin Artaud, Le théâtre et son double

\section{Introduction}

DE PRIME ABORD, PARLER D'UN THÉÂTRE AFRICAIN paraît relever de l'oxymore assuré, tant cette discipline semble avoir été forgée par les soins de l'Occident. C'est en tout cas le constat dressé par Robert Cornevin dans son Théâtre en Afrique noire et à Madagascar ${ }^{1}$, ouvrage où est faite l'apologie d'une prétendue politique de promotion du théâtre qu'aurait instiguée la colonisation française en Afrique. Pourtant, le théâtre semble être ancré dans les pratiques traditionnelles communautaires du passé des pays africains et l'arrivée des colonisateurs ne ravivera nullement l'ébahissement d'une telle découverte.

De par l'histoire de l'homme noir et du continent, le théâtre africain est naturellement traversé par un souci politique constant et ce n'est point ce que les critiques appellent le désenchantement du lendemain des indépendances qui prouvera le contraire. Le théâtre en Afrique aura toujours su garder, ne serait-ce qu'en filigrane, ce brin de rébellion, souvenir d'une blessure mal cicatrisée, malgré la succession des générations de dramaturges. Par conséquent, le théâtre africain ne peut-il être que politique ? Telle est la question que semblent poser les différentes métamorphoses d'un théâtre qui s'est évertué à sortir de l'état d'anesthésie et d'apathie dans lesquels la colonisation a laissé le continent. Au lendemain de la décolonisation des pays de l’Afrique noire, un hiatus colossal semble être dressé entre l'Afrique et le temps, qui dresse un portrait apocalyptique de l'avenir de ce qui est considéré, paradoxalement, comme le berceau de l'humanité. 
Ce souci du politique traverse de long en large l'histoire du théâtre africain. Cette suspension du temps dans le continent transparaît dans les créations dramatiques au lendemain des indépendances. Les dramaturges des années soixante appuient leur théâtre par un recours quasisystématique à l'histoire. Tout comme les poètes et dramaturges français de l'entre-deuxguerres qui, comme Jean Giraudoux, Jean Anouilh ou Louis Aragon, auront puisé dans la gloire du passé gréco-romain matière à résistance au fanatisme nazi, les dramaturges africains auront tenté d'exploiter leur passé comme un outil de réhabilitation du présent. Toute une génération brandira la gloire de Chaka Zulu comme on brandit un manifeste, dont Léopold Sédar avec son poème « Chaka » placé au centre des Éthiopiques, Senghor Tchicaya U Tam'si avec son Zulu (1977), Seydou Badian avec La mort de Chaka (1962), ou encore Condetto Nenekhaly-Camara avec Amazoulou (1970), et Abdou Anta $\mathrm{Ka}$, «l'homme de la rue » comme il se définit lui-même, avec les Amazoulou (1972). D’autres héros ont servi de modèle à la résistance à la conquête coloniale comme le damel ${ }^{2}$ Teigne Lat Dior, héros des Derniers jours de Lat Dior (1965) d’Amadou Cissé Dia, et du Procès de Lat Dior (1972) de Mbengue Mamadou Seyni, ou Ali Boury Ndiaye, roi du Djolof qui s'opposera à la résistance française dans L'exil d'Alboury (1967) de Cheik Aliou Ndao.

Des décennies plus tard, rien n'a changé, ou presque. Si la référence à l'histoire et au politique est clairement mise en avant dès les titres des pièces des années soixante, ce n'est ni la désillusion de l'après-indépendance des années soixante-dix et quatre-vingt et l'émergence d'un théâtre axé sur l'actualité et la critique des régimes totalitaires et sanguinaires instaurés, ni même la nouvelle génération des dramaturges des années quatre-vingt-dix qui réclament le contemporain, qui dérogera à la règle. Pourtant, l'entreprise ne sera plus jamais la même. Si dès Sony Labou Tansi, on quitte le territoire de l'identité noire à la Césaire, les préoccupations du théâtre contemporain africain restent cependant très politiques. C'est le cas de Caya Makhélé qui dans La Fable $d u$ cloître aux cimetières (1991) relève, à travers son personnage Makiadi, la question de la vacuité du sujet noir qui s'est toujours défini en opposition au blanc. C'est aussi le souci de Koffi Kawhulé dans Big Shoot (2006) où un personnage assujettit un autre qui se tient debout devant lui, celui-ci acceptant une domination où il ne s'agit ni plus ni moins que de lui inventer un nom, une histoire, une personnalité et des souvenirs - le même thème, avec des variations sur l'exil, est déjà abordé dans Village fou ou les Déconnards (2000) du même auteur. Le théâtre contemporain, tout en souhaitant délaisser le combat frontal avec l'ancien colonisateur, en aspirant à l'universel, continue de relever des questionnements liés au postcolonial comme l'identi- 
té fragmentée dans L'entre-deux rêves de Pitagaba (2000) de Kossi Efoui ou L'Exilé (2002) de Marcel Zang. Cette étude tentera d'examiner le développement des formes de protestation qui traversent le politique dans le théâtre africain et de voir dans quelle mesure les dramaturges contemporains - tout en s'inscrivant dans la lignée des précurseurs des années soixante - auront réussi à restituer au théâtre sa configuration première, celle d'une esthétique du spectaculaire et du dramatique, tout en sachant façonner le tout dans un moule implicitement politique. Il s'agira aussi de voir dans quelle mesure le théâtre contemporain africain relève les problématiques de la théorie postcoloniale en essayant, à travers une esthétique identitaire chaotique, d'inscrire l'Afrique au cœur des mouvances universelles et des préoccupations dramatiques actuelles.

\section{Crise de naissance}

Afin de comprendre le pourquoi d'une telle prépondérance du politique, il faudra essayer de répondre à la question suivante : le théâtre africain a-t-il besoin de se démarquer, ontologiquement et suivant un paradigme identitaire, du théâtre proposé par l'Occident ? Afin d'y répondre, il faudra revenir à l'origine de la naissance du théâtre à l'occidentale dans la scène négroafricaine. Il faut noter que l'arrivée du théâtre en Afrique ressemble plus à un débarquement qu'à autre chose et qu'il est d'emblée instrumentalisé par les forces de la colonisation. C'est l'École normale William-Ponty qui se chargera de divulguer un savoir et une pratique liés au théâtre, qui n'étaient en vérité qu'une manière détournée d'offrir aux yeux du spectateur occidental comme de l'Africain une image stigmatisée d'un théâtre folklorique truffé de culturalisme, et ce depuis la nomination - celui-ci étant appelé « théâtre indigène $\gg$. L'importance du théâtre à l'École ne se limitait pas aux simples exercice ou activité parascolaires. Il s'agissait d'un devoir parallèle à l'enseignement qui y était dispensé. Or, l'essentiel même de cet exercice montrait à quel point il s'agissait d'une tentative de l'enseignement occidental de réduire à l'état d'ornement et de divertissement exotiques ce qui définit l'homme africain transposé au théâtre. Créé par et pour des Noirs, ce théâtre révélait la stigmatisation qui traverse le premier théâtre africain dans sa configuration occidentale. En effet, il s'agissait pour les normaliens d'aller se documenter auprès des « sages » du village afin de se renseigner sur les traditions, coutumes et légendes de leurs milieux d'origine dans le but de les présenter sous forme d'une pièce de théâtre dont ils étaient à la fois acteurs, auteurs et metteurs en scène. Ces pièces étaient présentées en français à l'occasion de ce qui 
était appelé « la fête d'art indigène ». Si, de prime abord, le fait peut sembler noble, compte tenu de l'intérêt, en apparence, pour la spécificité de l'homme africain et ce qui fait la particularité de son quotidien, il s'agit surtout d'une manière de présenter l'art noir comme un condensé folklorique et de montrer l'homme africain comme un être incapable de participer à la réflexion aux grandes questions dont le théâtre pouvait être le véhicule. Les années trente sont une période faste pour le théâtre négro-africain (dit « pontin ») d'expression française qui y atteint son zénith - dans la configuration de la colonisation évidemment. C'est en 1937, au Théâtre des Champs-Élysées, que deux pièces seront jouées par les Dahoméens (du Bénin d'aujourd’hui) et les Ivoiriens dans le cadre de l'Exposition coloniale à Paris. Le rôle des Expositions étant bien évidemment de montrer aux habitants de la métropole ce qu'ils ignorent des colonies, il s'agissait logiquement d'exposer ce qui devait justifier encore plus la mission civilisatrice et les sacrifices qui l'accompagnaient - et qui n'étaient pas sans conséquence sur la métropole. Le théâtre pontin était non seulement, donc, le support qui donnait des colonies l'image d'une entité barbare baignée dans de vieilles pratiques superstitieuses, mais aussi l'occasion de voir un théâtre sous l'emprise de l'administration coloniale et - à travers le déplacement même des normaliens à Paris - d'une centralité dominatrice.

\section{La tragédie du roi Christophe, tragédie de l'Afrique}

Il est donc clair que la première rencontre de l'homme noir avec le théâtre du colonisateur était réduite à une vulgaire exposition d'une série de particularités culturalistes doublée d'un soubassement de domination. Les intellectuels noirs, dont Aimé Césaire, en saisiront rapidement toute la portée et ne tarderont pas à réagir. Si Césaire est un écrivain antillais, il semble, avec sa Tragédie du roi Christophe (1963), être l'instigateur d'une tradition qui va durer plus d'une décennie. Dès le titre, Césaire inscrit sa pièce dans le paradigme du théâtre occidental ; mieux, il lui choisit ce qu'il y a de plus familier pour un Occidental : une tragédie. Il ne déroge ensuite pas à la règle aristotélicienne en choisissant, de surcroît, un personnage de haut rang, un roi. Puis, dès la première scène, un commentateur, dont les apparitions vont rythmer la pièce, apparait. Commentant, récapitulant, offrant des précisions d'ordre historique, ce personnage renforce l'impression générale d'une pièce classique. Un premier coup d'œil donnerait l'impression d'une pièce qui s'inscrit dans la continuité du théâtre pontin et ce dès le prologue où est montrée l'une des activités festives favorites des Haïtiens, le combat de coqs - mettant donc en 
avant un élément du folklore noir ambiant. Pourtant, malgré toutes les similitudes apparentes de la pièce avec ce qui fait l'essence du théâtre classique occidental, Césaire se démarque en transformant cet épisode, qui devait être au départ anodin, en une satire politique qui ne sera pas sans inspirer un grand nombre de dramaturges africains par la suite. Césaire mêle à ce « semblant de tragédie » toutes sortes de registres, à l'instar du premier acte où burlesque et bouffonneries se joignent au tragique. L'auteur rompt, aussi, la fidélité à la tragédie classique doublement. D'abord, l'histoire s'invite insidieusement dans la pièce qui se transforme brusquement en un drame historique, sorte de tragi-comédie à la Corneille. Le combat de coqs sert de tremplin à une satire des politiques naissantes avec l'indépendance, et non à la monstration d'une quelconque psychologie comme c'est le cas dans les tragédies classiques. Mais, surtout, Césaire, à travers ce respect anachronique de quelques-unes des composantes de la tragédie classique, détrompe définitivement le spectateur en mettant en scène la déchéance d'un roi noir, personnage que la nature disqualifie, par définition (dans l'optique coloniale), de la noblesse du statut de héros tragique. Le choix de la tragédie permet à Césaire de mettre l'accent sur la déchéance politique qui suit les indépendances et de présenter l'abus d'autorité des hommes politiques des pays fraîchement décolonisés comme une fatalité. L'auteur, en mariant au sein d'une même pièce un format tragique occidental et l'histoire d'un héros de la libération du joug colonial de ce même Occident, semble se prononcer en faveur d'un combat syncrétique contre la tyrannie et la domination dans le monde. Il l'exprime lui-même dans Présence Africaine :

Qu'on le sache: en articulant notre effort sur l'effort de libération des peuples colonisés, en combattant pour la dignité de nos peuples, pour leur vérité et pour leur reconnaissance, c'est en définitive pour le monde tout entier que nous combattons et pour le libérer de la tyrannie, de la haine et du fanatisme ${ }^{3}$.

\section{Théâtre historique, théâtre épique}

Si c'est avec le mouvement de la négritude qu'est vraiment lancée une lutte organisée pour l'officialisation et la reconnaissance de la littérature africaine, force est de constater que cette quête s'effectue en parallèle d'un combat mené sur fond identitaire. L'efficacité du procédé littéraire de La tragédie du roi Christophe 4 comme décrite par Liliyan Kesteloot - malgré toute la controverse ${ }^{5}$ qui peut accompagner le nom de cette spécialiste - est parfaitement perçue au continent africain où une multitude d'auteurs se saisissent de l'histoire et du sort tragique des héros de la résistance au colonisateur et 
des guerres des libérations comme d'un outil d'éclairage de la période coloniale et de ses travers, cette fois du point de vue de l'Africain. La focalisation deviendra définitivement interne, doublée d'un optimisme qui s'étalera sur toute une décennie. Il faut dire que c'est encore à une autre figure de proue de la négritude, le poète-président Léopold Sédar Senghor, que l'on doit cette réhabilitation des héros du passé colonial, lui qui a placé son poème « Chaka » au cœur de son recueil Les Éthiopiques. Force est de constater aussi que ce poème se donne à lire comme un dialogue théâtral, le poète le définissant comme un « poème dramatique à plusieurs voix » entre Chaka et une Voix Blanche. Celle-ci interpelle le héros en ces termes : « Ma parole Chaka, tu es poète... ou beau parleur... un politicien ${ }^{6}$ ! $\gg$ De là, tout un théâtre historique - et forcément politique en conséquence - verra le jour. Une longue liste des héros de l'ère coloniale seront réhabilités, et deviendront arguments d'autorité contre une histoire écrite par l'homme blanc, preuve irréfutable d'une Afrique qui n'acquiesce nullement à la transplantation de la civilisation occidentale sur ce qui est soi-disant un continent sauvage. Partant de là, tout un édifice s'érigera sur cette pierre qu'aura lancée Senghor, inspiré luimême par le roman de Thomas Mfolo Chaka paru en 1939. Le personnage de Chaka Zulu inspirera toute une génération d'auteurs qui en feront la figure de l'Africain insoumis et révolté. À la différence de La Mort de Chaka de Saydou Badian Kouyaté publiée en 1962, la plupart des pièces qui mettent en scène la vie de Chaka sont postérieures à La Tragédie du roi Christophe. La pièce de Badian suit presque la même logique que celle de Césaire, mettant elle aussi en scène un héros qui régnera sur son peuple dans un bain de sang. La pièce se construit en cinq actes et annonce elle aussi dès son titre le tragique de la destinée du héros. La plupart des pièces qui exploitent le personnage historique de Chaka contiennent dès le titre des références qui sont indispensables à la mise en connexion avec l'histoire et le politique. Quelques-unes affichent en gras le nom du héros comme le Chaka (1971) de Djibril Tamsir Niane et La Mort de Chaka de Seydou Badian. D'autres signalent l'appartenance ethnique du héros comme le très didactique Amazoulou de Condetto Nenekhaly-Camara, Les Amazoulou d'Abdou Anta-Ka ou le Zulu de Tchicaya U Tams'i. Les pièces mettent une à une l'accent soit sur le peuple tyrannisé soit sur le personnage de Chaka dont il s'agira de grossir l'ambition débordante et le vœu ardent de dictature. Pourtant, toutes les pièces s'appuient sur l'histoire comme élément d'affirmation et de reconnaissance de l'identité noire. Il s'agit aussi d'une confirmation du bien-fondé de l'histoire africaine, d'un outil de critique politique et de mise en garde contre ce qui pourrait devenir un néo-colonialisme des nouveaux dirigeants africains. 
Le choix de Chaka Zulu est pertinent s'il se conçoit dans une mesure analogique parallèle à l'histoire du continent. En effet, le parcours du personnage bâtard, ayant difficilement accédé au trône et bataillant sans cesse pour sa sauvegarde, ressemble étrangement à l'histoire de l'Afrique dont l'identité fut longtemps stigmatisée et dont l'indépendance promet un combat encore plus violent contre ses nouveaux gouverneurs. Par conséquent, ce n'est pas seulement l'occasion d'une quête de reconnaissance de l'histoire noire mais aussi une tentative de faire-valoir celle-ci en l'intégrant comme une composante essentielle de l'histoire universelle, tel que l'affirme l'historien Joseph Ki-Zerbo, rapporté par Midiohouan : «Tchaka est l'un des plus grands conquérants de l'histoire de l'Afrique, et son nom mérite d'être retenu par l'histoire universelle ${ }^{7}$. »

Pourtant, affirmer que cette exploitation de l'histoire conduit à faire du théâtre historique serait manquer ce qui fait sa force. Il s'agit, d'abord, d'un théâtre politique, ces personnages et ces événements historiques servant de prétexte à la jubilation et la fête. La plupart de ces dramaturgies ne visent pas l'exactitude historique. Cheikh Aliou Ndao ne conçoit pas ses pièces comme des thèses historiques. Il mélange volontairement une multitude de personnages fictifs qui côtoient des personnages qui ont vraiment existé. C'est le cas de L'exil d'Alboury qui raconte l'histoire d'Ali Bouri Ndiaye, dit Alboury, neveu de Lat Dior, ou même du Fils de l'Almany (1973) qui relate les périples de Samory Touré ${ }^{8}$, personnage à qui le Malien Massa Makan Diabaté a consacré deux pièces : Une si belle leçon de patience (1973) et la bien tardive Une hyène à jeu (1988). Il s'agit, ensuite, d'un théâtre épique. En effet, la plupart des productions dramatiques du lendemain des indépendances renouvellent l'héritage du théâtre pontin tout en meublant le vide folklorique qui l'habitait. Plusieurs des éléments du théâtre épique, tel que défini par Patrice Pavis ${ }^{9}$, s'y retrouvent. En opposition au dramatique, celui-ci se caractérise par une reconstitution du passé à travers une exposition des événements qui forment une totalité. Les personnages historiques sont donc exploités afin d'exalter l'identité noire qui se trouve non seulement reconnue, mais aussi fêtée et galvanisée. Chaka n'est pas le seul personnage historique à se retrouver au centre de ce mouvement dramatique mais aussi Lat Dior comme dans Les Derniers jours de Lat Dior d'Amadou Cissé Dia ou Le Procès de Lat Dior de Mbengue Mamadou Seyni, des titres mettant encore l'accent sur le tragique de la situation du héros, dont seuls les derniers jours sont gardés. Jean Pliya mettra en scène Gbêhanzin (ou Bêhanzin) dans ce qui deviendra un classique de la littérature négro-africaine d'expression française, Kondo le requin (1966). D'autres récits lient intimement le théâtre avec le politique, servant de sup- 
port au récit de la résistance des peuples noirs aux interventions étrangères. C'est le cas des deux pièces consacrées à Lat Dior, mais aussi à celles qui relatent les périples de son neveu Alboury, ou encore du Sissako ou la dernière Citadelle (1971) de Djibril Tamsir Niane. La résistance, quand elle ne cible pas le colonisateur, s'attaque à toute perversion de l'intégralité des traditions africaines, comme avec Tarentelle noire et diable blanc (1976) de Sylvain Bemba où Faustino - dont l'onomastique annonce subtilement la référence occidentale -, commerçant blanc achète les âmes des noirs qui veulent bien les lui vendre. L'exemple de Béatrice du Congo (1970) de Bernard Dadié porte aussi une autre exploitation politique qui décrit la résistance de Dona Béatrice, une prophétesse qui perçoit derrière la coopération établie avec les Bitandais un grand projet de colonisation du Zaïre, dans une mise en abyme des méfaits despotiques de la coopération franco-africaine. Parallèlement à ces exemples qui puisent dans l'histoire de la résistance à la colonisation, l'histoire immédiate s'invite dans le théâtre africain comme dans l'œuvre d'Alexandre Kum'a Ndumbe III à travers Amilcar Cabral ou la tempête en Guinée-Bissau (1976), écrit en hommage au héros de la guerre d'indépendance du pays, mort assassiné, ou Kafra-Biatanga (1973) qui évoque les événements sanguinaires du Biafra au Nigeria et de Katanga au Congo.

\section{Intervenir sur l' histoire en train de se faire}

Il faut dire que le théâtre historique africain ne fut, jusque-là, que politique. L'utilisation que les auteurs tendent à tirer des épisodes, des personnages et des événements historiques de l'Afrique sert à mettre en évidence l'attachement du présent chaotique au passé. Mais elle sert aussi à puiser dans ce même passé les raisons de ce chaos ainsi que les fragments d'une identité noire que le colonisateur s'est efforcé d'effriter. Seule une mythification de l'héroïsme noir et de ses figures de proue saurait sensibiliser l'Africain quant à ce qui fut son histoire et l'essentiel de son identité. Les indépendances n'ont pas libéré les écrivains africains de leur devoir d'histoire. Cette période faste, où il sera question de chanter la liberté, ne sera que trop brève. Elle durera moins de dix ans. Au lendemain des décolonisations, les auteurs africains se rendront vite à l'évidence et le « soleil des indépendances » se couchera aussi vite qu'il s'était levé. C'est le désenchantement. Aussi le théâtre devient-il le support de la dénonciation, les dramaturges souhaitant désormais intervenir directement sur le présent, sur l'histoire en train de se faire. L'histoire immédiate viendra se greffer au théâtre qui deviendra résolument politique et social. Césaire encore lui - annonçait déjà dans Une saison au Congo (1966) les prémices 
d'un néocolonialisme naissant, fruit des collaborations internes avec le colonisateur ${ }^{10}$. D'une manière plus explicite, Bernard Dadié dresse dans Monsieur Thôgô-Gnini (1970) - dont le nom et les caractéristiques rappellent étrangement Monsieur Jourdain - le portrait d'un parvenu cupide et foncièrement immoral qui aspire, à travers l'argent, à dominer ses semblables à l'image de Théobald Ngandou, l'usurier, de L'homme qui tua le crocodile (1972) de Sylvain Bemba ou de Pérono de Je soussigné cardiaque (1977) de Sony Labou Tansi. D’autres auteurs s'attaqueront aux hommes politiques africains de leur époque, dénonçant tour à tour leur dictature et grossissant leurs travers. Dans la veine du roman de Yambo Oulouguem, le Devoir de violence (1968), primé par le Renaudot, des pièces comme Le train spécial de son Excellence (1978) de Guillaume Oyono-Mbia, Termites (1976) d'Eugène Dervain ou La secrétaire particulière (1973) de Jean Pliya démystifient le mythe de l'innocence des chefs africains - l'intelligentsia de la négritude ayant, jusqu'aux années soixante-dix, éloigné toute responsabilité des chefs africains devant le colonisateur et donc l'impossibilité que ceux-ci aient conclu une quelconque félonie ${ }^{11}$. La seule tentative d'Ahamadou Kourouma au théâtre, Tougnantigui ou le diseur de vérité écrite en 1972, lui valut vingt ans d'exil, seulement après quelques représentations à Abidjan. Un peu à l'image des drames historiques d'après-indépendance, la pièce de Kourouma met en scène un acteur principal de la résistance au colonisateur, Diarra, qui à travers cette lutte accédera au pouvoir absolu. L'enjeu de la pièce repose sur le paradoxe du titre, chose qui fait dire à Laura Menéndez-Pidal Sendrail que « ce personnage rassemble les points communs aux dictateurs africains. Sa particularité littéraire retombe sur sa dénomination de Diseur de vérité qui reprend le titre de la pièce ${ }^{12} \gg$. En effet, Diarra ressemble étrangement à tous les héros de l'histoire de l'Afrique qui se sont transformés, bon gré mal gré, en (mauvais) pères de la nation. Et c'est à travers le discours que cette «douce » domination s'effectue car «Diarra est un véritable manipulateur de la parole. Il transforme constamment la vérité en fin connaisseur de l'âme humaine et c'est à travers le mensonge qu'il exprime sa vérité ${ }^{13} \gg$. Dans la même optique le drame satirique Le Président (1970) de Maxime N’Débéka, figure de proue des manifestations contre le pouvoir au Congo Brazzaville des années 1980, lui vaudra l'exil en France ainsi que la condamnation à mort ${ }^{14}$. On retrouve la même agressivité dans les pièces de Tchicaya U Tam'si Le destin glorieux du Maréchal Nnikon Nniku Prince qu'on sort (1970) qui, à l'instar de son Zulu, dessine la dictature comme un cercle vicieux, ou de Bernard Dadié qui avait déjà anticipé le désenchantement en 1969 avec Les voix dans le vent. 
Il est donc clair que la récupération de la théâtralité occidentale dans les dramaturgies africaines d'expression française ont affiché un souci politique continu, fruit du constat apocalyptique des sociétés africaines postindépendantes. Un théâtre social accompagne aussi le politique et l'historique à travers des pièces qui mettent en scène les travers des sociétés naissantes. Guillaume Oyono Mbia avec ses Trois prétendants, un mari (1964), Jusqu'à nouvel avis (1969) ou Notre fille ne se mariera pas (1971), Ferdinand Mouangassa avec N’Ganga Mayala (1976), Sylvain Bemba avec Une eau dormante (1972), Alexandre Kuma N’Dumbe III avec Lisa, la putain de... (1976) ou bien tardivement Amadou Koné avec Le respect des morts (1980) s'intéressent tout particulièrement aux habitudes et normes du social de leurs milieux. Mais contrairement au théâtre pontin, les nouveaux dramaturges africains prônent des sociétés évoluées à la fois modernes et authentiques. Cependant, le traitement du social qu'adoptent ces nouvelles dramaturgies ne se déconnecte jamais du politique qu'elles gardent dans le collimateur étant, bien évidemment, à la fois la cause de la stagnation de ces sociétés, mais aussi le moteur par lequel elles peuvent décoller.

\section{Les années 1990 : penser le contemporain}

Cependant, tout ce théâtre reste profondément lié à des questionnements sociaux et identitaires qui, dans plupart des cas et à quelques exceptions près, évacuent la question esthétique, et se désynchronisent du reste des productions dramatiques contemporaines qui, désormais, défont jusqu'à la moelle osseuse l'essence de ce qui est - ou fut - théâtre. Car les indépendances en Afrique donnent l'impression qu'un continent s'est réveillé d'un sommeil léthargique, sorte de coma qui dresse un voile spatio-temporel insurmontable, entre elle et le monde. Plusieurs penseurs comme Cornevin ou Alain Ricard dans son interminable débat avec Ngugi Wa Thiong'o, et plus tard de celui-ci avec Ebrahim Hussein ${ }^{15}$, ont épuisé la question de l'existence ou non du théâtre dans le continent, les uns affirmant l'absence de théâtre africain et que seules des formes rituelles existaient, alors que d'autres s'acharnent à prouver à tout prix son existence et même à en fonder une théorie et une pratique, comme le «théâtre-rituel » de Marie-José Hourantier qui pense que « le théâtre, mot dont l'étymologie grecque révèle une profonde appartenance à l'Occident, ne peut satisfaire un public africain ${ }^{16} \gg$. Pourtant, ces débats font l'économie d'une considération de taille qui leur est, néanmoins, contemporaine. Celle-ci souhaite que le théâtre ne soit plus défini comme une entité inchangeable qui aurait caracolé depuis l'Antiquité jusqu'à nos 
jours sous une seule et unique forme. Car il n'est plus question de parler d'un théâtre au singulier mais des théâtres, ce que tout le volet performatif et spectaculaire des nouvelles formes contemporaines ne cesse de crier. Néanmoins, et dès les années quatre-vingt-dix, une nouvelle génération de dramaturges va éclore, amorcée par le subversif Sony Labou Tansi, reconnu comme l'instigateur de toute une dramaturgie qui désire s'inscrire dans le contemporain. John Conteh Morgan, rapporté par Sylvie Ndome $\operatorname{Nguilla}^{17}$, détecte deux grandes tendances radicales dans les productions avant-gardistes du théatre africain, l'une tournée vers un militantisme politique et l'autre vers une radicalité esthétique qui incorpore mythes, musique, danse et chant. Ces deux directions montrent que le théâtre africain d'expression française (et africain en général) n'est pas prêt de quitter le terrain du politique, même avec des tentatives comme celle d'Hourantier, qui ne sont qu'une réaction volte-face à l'encontre du théâtre occidental. Tchicaya U Tam'si, lui, conçoit la chose autrement. Car, pour lui, « l'Afrique noire de l'oralité n'a pas la même conception du théâtre que l'Europe des lettrés. Il fallait simplement dire cela et ne pas décréter, comme cela a été fait, que la notion même de théâtre est étrangère au génie de l'Africain ${ }^{18} \gg$.

Toute une génération d'auteurs se retrouve, dès les années quatre-vingtdix, dans un entre-deux identitaire, favorisé par un exil qui les parachute directement en métropole. Sony Labou Tansi est le premier à s'être consacré à « une dramaturgie de l'audace $[. .$.$] qui ose brouiller les repères et décons-$

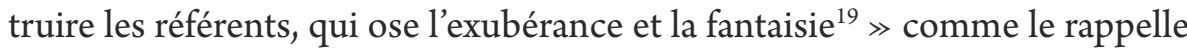
Sylvie Chalaye. Avec lui s'opère un changement radical qui tranche définitivement avec les écritures dramatiques africaines qui ont précédé et une nouvelle ère de questionnements s'ouvre. De nouvelles approches textuelles aspirent à battre en brèche à la fois le théâtre dans son acceptation aristotélicienne mais aussi à abandonner ce qui a pendant longtemps contraint les auteurs africains à des dramaturgies de la contestation pure et simple. On quitte avec ces auteurs le territoire de la recherche d'une identité intégrale décelée dans les méandres du folklore noir, d'une Afrique coupée du temps et du reste du monde, image parfaite d'un continent immuable qui rappelle les récits de voyage européens du XIX ${ }^{\mathrm{e}}$ siècle. Car ces auteurs refusent ce que Koffi Kwahulé appelle une « quête de compensation ${ }^{20}$ ». En d'autres termes, il ne s'agit plus de chercher à compenser le présent par un passé glorieux, mais de dresser un état des lieux fidèle de la situation en Afrique qui doit désormais être sur le diapason de l'universel et ce qui se produit dans le reste du monde. C'est dans cette optique que Kossi Efoui écrit Le Carrefour (1990) où il se livre à une expérimentation métadramatique à la Pirandello. Or, 
ses personnages cherchent à se défaire de leur statut au lieu de l'embrasser comme dans la pièce italienne. Efoui prolonge cette réflexion dans le «cycle du carrefour » avec deux autres pièces, La Malaventure (1993) et Que la terre vous soit légère (1995) où les mêmes trois personnages désirent échapper à l'emprise de l'auteur et de l'identité dramatique qui leur est imposée. Cette pièce propose, selon Sylvie Chalaye, une nouvelle façon de penser le théâtre en Afrique ${ }^{21}$. Caya Makhélé, lui, désire dans La Fable du cloître des cimetières (1995) brouiller l'espace-temps à travers l'épopée de son personnage Makiadi, inscrivant le théâtre africain dans une topographie qui n’a jamais été la sienne. Désormais, le souci du politique ne quittera plus jamais ce théâtre à qui Ndomé Nguilla donne le qualificatif « chaotique ${ }^{22}$ ». La plupart de ces dramaturgies dressent un portait défiguré d'une Afrique déchirée qui n'arrive toujours pas à cicatriser la blessure coloniale. Toutes les pièces, ou presque, relèvent, différemment certes, les mêmes questionnements identitaires de leurs prédécesseurs. Car en refusant d'adhérer à la définition d'une identité noire qu'il faut à tout prix rechercher et en mettant en scène des personnages vagabonds dont l'identité est un grand amas pulvérisé, le politique ne fait que s'inverser, devenant constat alors qu'il était recherche immédiate de solution. C'est le cas du personnage errant de Makhélé dans La Fable du cloître des cimetières ou du personnage de Big Shoot de Kwahulé ou Village fou ou les Déconnards du même auteur qui met en scène un personnage vivant isolé dans une mansarde où il reçoit la visite de ses ancêtres. Efoui met aussi en scène l'errance d'une boxeuse dans L'entre-deux rêves de Pitagaba conté sur les trottoirs de la radio, thème que reprend Makhélé dans Destins (2009) ou Kwahulé avec Cette vieille magie noire (1993). Marcel Zang dans L'Exilé exprime l'émiettement identitaire et l'abandon définitif de sa quête à travers cette réplique d'Imago :

Je veux bien reconnaître certains liens avec l'Afrique [... ] mais mon identité avec l'Afrique est une identité parmi d'autres, parmi des centaines d'autres qui n'ont rien à voir avec l'Afrique.

Avant de finir sur un constat des plus parlants :

et à supposer que j'aie une identité, ce ne peut être que cette somme d'identités diverses $^{23}$. 


\section{Conclusion}

En définitive, le théâtre africain reste en grande majorité fondamentalement attaché au politique. Il s'agit d'une manière ou d'une autre pour ce théâtre de continuer à exister car, pour une dramaturgie fraîchement créée, la quête de reconnaissance n'est que continue. Car, pour Kwahulé, «c'est maintenant que le plus difficile commence, maintenant qu'il ne s'agit plus d'inventer le théâtre mais de le créer ${ }^{24} \gg$. Le théâtre tout aussi bien que les spectacles africains restent politiques, car nés dans et pour la polis. L'oralité des pratiques traditionnelles fabrique un théâtre ancré dans la cité qui, même quand il revendique le mondial, garde cette oralité qui lui est propre comme dans «l'écriture-jazz ${ }^{25} \gg$ de Kwahulé. Le théâtre et la poésie africains n'ont été jusqu'à aujourd'hui que politiques, et n'y a-t-il que les noms de Tchicaya U Tam'si et Sony Labou Tansi pour l'illustrer - U Tam'si et Tansi signifiant en langue bantoue « la petite feuille qui parle pour son pays. » Pour U Tam'si « il n'y a pas de peuple qui ne théâtralise le moindre des actes essentiels de sa $v^{26}{ }^{26}$. $\gg$ Or, si le théâtre puise dans la vie quotidienne des sociétés africaines et leurs réalités, il finit par embrasser leurs revendications et leurs aspirations. Même quand les dramaturges de la diaspora africaine veulent inscrire l'Afrique dans le vaste panorama des productions théâtrales contemporaines, le fantôme du politique se profile à l'horizon et le besoin de reconnaissance à l'échelle mondiale se fait pressant pour le continent.

La métaphore de la boxe qui traverse l'œuvre de plusieurs dramaturges contemporains africains semble résumer notre propos ici. Car le continent africain ne peut aujourd'hui produire qu'un théâtre qui doit « boxer la situation », pour reprendre les termes de Dieudonné Niangouna, comme il l'écrit dans M'appelle Mohammed Ali (2014). Car le théâtre africain doit lutter sur plusieurs fronts. À l'extérieur, il doit combattre la politique néocolonialiste de la grande « république mondiale des lettres ${ }^{27}$ », pour reprendre le titre de l'ouvrage de Pascale Casanova, à l'instar de la programmation du Festival d'Avignon 2017 qui fait dire à Niangouna qu'« inviter un continent sans sa parole c'est inviter un $\operatorname{mort}^{28} \gg$. À l'intérieur, la lutte se fait contre une pensée réfractaire qui refuse d'encourager le théâtre à l'image de la censure qui frappe Niangouna au Congo Brazzaville. Le lourd passé colonial a créé chez l'Africain, et l'homme noir en général, un réflexe d'auto-défense vis-à-vis de tout ce qui provient de la métropole occidentale. Il y est allé, à un moment précis de l'histoire, du bon comme du mauvais. À l'attitude de domination à laquelle dut faire face l'homme noir, au vouloir de soumission qui fut longtemps le projet de l'Europe blanche, répond automatiquement à la fois une 
attitude d'insurrection et de résistance de la part du colonisé mais, aussi, la création d'un certain automatisme fanatique chez celui-ci, qui est le corollaire de l'extrémisme blanc chez le colonisateur.

Car en Afrique, il faut d'abord trouver un public de théâtre. Étienne Minoungou, jeune dramaturge burkinabé, créateur des «Récréâtrales» pense que «le problème vient du fait que le théâtre africain n'a pas de soutien public. Et il peine à créer un véritable public $c^{29} \gg$. Le théâtre en Afrique ne jouit toujours pas du soutien du public et reste la lourde besogne de quelques troupes qui ont le plus grand mal à subsister. Si le théâtre populaire est cependant bien diffusé dans les pays africains, il reste prisonnier d'une localité asphyxiante. Le geste des dramaturges du théâtre contemporain africain de la génération des années quatre-vingt-dix est un acte ambitieux, certes. Mais ce geste l'est dangereusement, car en voulant s'intégrer à la cartographie des grandes œuvres contemporaines, le passage par les instances de légitimation des centres littéraires devient nécessaire et ces mêmes auteurs tombent dans ce qu'ils reprochaient à leurs prédécesseurs, c'est-à-dire un recours quasi systématique au mécénat occidental. À l'échelle nationale, la pratique théâtrale reste un choix difficile. La Nouvelle Tribune du Bénin ${ }^{30}$ rapporte que Tola Koukoui, metteur en scène béninois, ne put en 2016 payer ses comédiens en tournée qu'après l'intervention du président Patrice Talon qui lui commanda, providentiellement, le chef-d'œuvre de Jean Pliya Kondo le Requin dans une énième démonstration de l'emprise du pouvoir politique sur la vie théâtrale africaine. Koffi Kwahulé, en parlant de cette même édition 2017 du Festival d'Avignon, préfère à la programmation officielle du On, les balbutiements du Off. Il affirme que « dans le Off, on ne vient pas avec une salle déjà remplie, on doit la remplir, et c'est par mon travail que peu à peu je vais remplir la salle $^{31}$. $\gg$ Car le dramaturge ivoirien est bien conscient de tout le processus d'exposition du Festival et préfère l'ambition débordante des créateurs du Off qui « viennent là en mangeant de la vache enragée pour venir présenter des auteurs ou des pièces qu'ils ont conçu eux-mêmes, et c'est cette aventure du théâtre qui m'intéresse ${ }^{32} \gg$. Si le théâtre africain, par la richesse indéniable de ses productions, a jusque-là été réaction, une anagramme seulement l'empêche de devenir création. 
Transformation du politique dans le théâtre négro-africain d'expression française des débuts à nos jours

\section{Notes}

1. Robert Corvin, Le théâtre en Afrique noire et à Madagascar, Paris, Le Livre Africain, 1970.

2. Titre donné aux souverains du royaume historique du Cayor situé au Sénégal.

3. Aimé Césaire, "L'homme de culture et ses responsabilités " in Présence africaine, $\mathrm{n}^{\text {os }}$ 24-25, février-mai, 1959.

4. Lilyan Kesteloot, Histoire de la littérature négro-africaine, Paris, Karthala, 2001, p. 237.

5. Guy Ossito Midiohouan parle d'une « erreur largement divulguée par l'ouvrage de Kesteloot Les écrivains noirs de langue française : naissance d'une littérature » qui daterait la naissance de la littérature négro-africaine avec la mise en marche du mouvement de la négritude. Voir Guy Ossito Midiohouan, «Le théâtre négro-africain d'expression française depuis 1960 » in Mongo Beti et Odile Tobner (dir.), Peuples Noirs Peuples Africains, no 31, 1983, p. 55.

6. Léopold Sédar Senghor, Euvre poétique, Paris, Seuil, 1990 [1956], p. 122-137.

7. Joseph Ki-Zerbo, Histoire de l'Afrique noire : d'hier à demain, Paris, Hatier, 1972, p. 360.

8. Fondateur de l'empire Wassalou, il fut une grande figure de la résistance à la colonisation française. L'Ivoirien Bernard Zadi le mettra aussi en scène dans le drame historique Les Sofas (1979).

9. Patrice Pavis, Dictionnaire du théâtre, Paris, Éditions Sociales, 1980, p. 129-132.

10. Albert James Arnold parle d'un «Patrice Lumumba confronté aux mécanismes ainsi qu'aux manœuvres sournoises et bien rodées du néocolonialisme sur la scène du Congo fraîchement décolonisé ». James-Arnold Albert, Aimé Césaire : Poésie. Théâtre, Essais et Discours, Paris, CNRS, 2013, p. 1103.

11. Lilyan Kestellot, "La littérature négro-africaine face à l'histoire de l'Afrique » in Afrique contemporaine, 2012/1, n 241, p. 43-53.

12. Laura Sendail Menendez-Pidal, «La pièce oubliée d'Ahmadou Kourouma : Le diseur de vérité d'après une analyse postcoloniale » in Anales de Filología Francesa, n 21, 2013, p. 248.

13. Ibid.

14. « [Maxime N’Débéka] est aujourd'hui, avec Le Président, un dramaturge populaire dont l'efficacité s'unit au talent », quatrième de couverture du Président, Paris, L'Harmattan, « Encres Noires », 2000 [1970].

15. Cf. Alain Ricard, L'invention du théâtre. Le Théâtre et les comédiens en Afrique noire, Paris, L'Âge de l'homme, 1986. Ngugi Wa Thiong'o, Decolonizing the Mind, the Politics of language in Africa literature, Londres: J. Curry, 1986. Ebrahim Hussein, Comment écrire pour le théâtre en suivant Aristote?, trad. Par Kasoro Tumbwe, Närobi, CREDU, 1991.

16. Elle continue son argumentation en expliquant que «cette passivité du spectateur qui se contentait de "contempler" ne pouvait convenir aux représentations traditionnelles et à leur système de participation. Les tragédies grecques étaient fort éloignées d'un Kotéba ou d'un rituel d'Abyssa! » M.-J. Hourantier, Du Rituel au théâtre-rituel. Contribution à une esthétique théâtrale négro-africaine, Paris, L'Harmattan, 1984, p. 36.

17. John Conteh Morgan, "The Other Avant-Garde: The Theater of Radical Aesthetics and the Poetics and Politics of Performance in Contemporary Africa " in James M. Harding et John Rouse (dir.), Not The Other Avant-Garde, The University of Michigan 
Press, 2006, p. 92-124. Rapporté par Sylvie Nguila Ndome, Nouvelles dramaturgies africaines francophones $d u$ chaos, thèse de doctorat sous la direction de Maria Brewer et Sylvie Chalaye. https://conservancy.umn.edu/bitstream/handle/11299/171460/ NdomeNgilla_umn_0130E_15693.pdf, consultée le 6 janvier 2018.

18. Tchicaya U Tam'si, «Les origines sacrées de notre théâtre » in Le Monde diplomatique, décembre 1987, p. 23.

19. Sylvie Chalay, L'Afrique noire et son théâtre au tournant du XXe siècle, Rennes, Presses Universitaires de Rennes, « Plurial », 2001 p. 13.

20. L'expression est rapportée par Virigine Sourbier dans Le Théâtre de Koffi Kwahulé : L'utopie d'une écriture-jazz, Layde, Brill, 2014, p. 43.

21. Sylvie Chalay, «Le Théâtre de Kossi Efoui : une poétique du marronnage » in Africultures, no 86, novembre 2011, p. 8.

22. Sylvie Ndome Nguilla, Nouvelles dramaturgies africaines francophones du chaos, op. cit.

23. Marcel Zang, L'Exilé, Arles, Actes Sud, 2002, p. 30.

24. Koffi Kwahule, cité par Virginie Soubrier, Le Théâtre de Koffi Kwahulé : L’utopie d'une écriture-jazz, op. cit., p. 26.

25. Cf. Virginie Soubrier, Le Théâtre de Koffi Kwahulé : L'utopie d'une écriture-jazz, op. cit.

26. Tchicaya U Tam'si, «Les origines sacrées de notre théâtre » in Le Monde diplomatique, op. cit.

27. Voir l'indispensable ouvrage de Pascale Casanova, La République mondiale des lettres, Paris, Seuil, « Points », 1999.

28. Il exprimait aussi, sur sa page Facebook, son ahurissement de voir "zéro théâtre " africain au Festival d'Avignon 2017.

29. Interview avec Étienne Minoungou dans Demain le monde, no 11, janvier-février 2012. Consulté le 29 décembre 2017. https://www.cncd.be/Le-theatre-africain-dans-sa

30. Olivier Ribous, La Nouvelle Tribune, 31 mai 2016. https//lanouvelletribune.info/ archives/benin/28986-kondo-piecede-pliya-qui-a-fait-craquer-talon Consultée le 5 janvier 2018.

31. Koffi Kwahule, interview RFI du 15 juillet/2017. https://www.youtube.com/ watch?v=YC7z3gqAFi0 Consultée le 22 décembre 2017.

32. Ibid. 